\title{
原子簇 $\mathrm{C}_{n}$ 中碳原子的轨道杂化的研究 *
}

\author{
陈琼王成瑞刘道玉
}

(武汉大学化学系, 武汉 430072)

\section{关键词 原子簇、碳尞多面体、原子轨道杂化}

近年来, 质谱实验发现 $\mathrm{C}_{180}$ 能稳定存在 ${ }^{[1]}$, 理论推算得到 $\mathrm{C}_{180} \mathrm{C}_{540}, \cdots$ 具有 $I_{h}$ 对称性, 预测 $\mathrm{C}_{540}$ 也是一个稳定的结构 ${ }^{[2]}$, 但还未见它们的键长、键角、二面角等结构参数报道. 本文在研究 $\mathrm{C}_{60}$ 和 $\mathrm{C}_{70}$ 分子中碳原子的轨道杂化的基础上 ${ }^{[3,4]}$, 对具有 $I_{h}$ 对称性, 未知键长: 键角、二面角等 结构参数的碳原子簇 $\mathrm{C}_{180}, \mathrm{C}_{540}, \cdots, \mathrm{C}_{43740}$ 中碳原子的轨道杂化进行研究.

\section{1 近似公 式}

具有 $I_{h}$ 对称性的碳原子簇 $C_{n}$ 是由 12 个五边形和 $\left(\frac{1}{2} n-10\right)$ 个六边形构成的封闭多面 体, 其 $n=20 \times 3^{k}(k=0,1,2 . \cdots)^{[2]}$. 设五边形和六边形边长均为 $l$, 其角分别是 $2 \varnothing$ 和 $2 \omega$, 则 计算得到碳原子簇 $\mathrm{C}_{n}$ 的封闭多面体的面积是

$$
\lambda_{\ell_{n}}=[15(\operatorname{tg} \varnothing-\operatorname{tg} \omega)+0.75 n \operatorname{tg} \omega] l^{2} \text {. }
$$

图 1 是碳原子族 $\mathrm{C}_{n}$ 虚拟球, $o$ 为球心, $r_{n}$ 是球的半径. 第 $n$ 个碳原子置于球面 $n$ 的位置, 和 $n$ 直接相邻的三个碳原子 $i, j, k$ 分别处于球面 $i, j, k$ 位置, $n i, n j$ 和 $n k$ 的长皆为 $l$, 球的面 积是

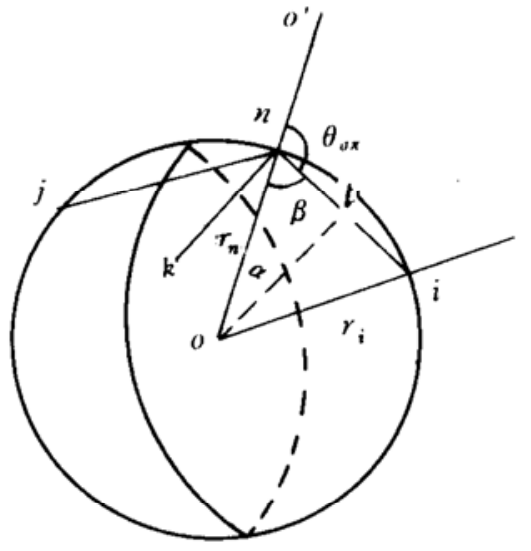

图1 原子簇 $\mathrm{C}_{n}$ 的虚拟球

1993-09-18 收稿, 1994-03-08 收修改稿.

* 国家自然科学基金资助项目.

$$
A_{n}=4 \pi r_{n}^{2}
$$

设 $A_{n}$ 近似等 于 $A_{C_{n}}$ 得到球的半径是

$$
r_{n}=\frac{l}{2 \sqrt{\pi}}[15(\operatorname{tg} \varnothing-\operatorname{tg} \omega)+0.75 n \operatorname{tg} \omega]^{1 / 2} .
$$

由几何学方法计算得到

$$
l=2 r_{n} \sin \left(\theta_{\sigma \pi}-90^{\circ}\right),
$$

将(3)式代人(4)式得到

$$
\cos \theta_{\sigma \pi}=-\left[\frac{\pi}{15(\operatorname{tg} \phi-\operatorname{tg} \omega)+0.75 n \operatorname{tg} \omega}\right]^{1 / 2},
$$


设 $\mathrm{C}_{n}$ 中第 $n$ 个碳原子的四个杂化轨道分别是 $\psi_{1}, \psi_{2}, \psi_{3}$ 和 $\psi_{4}$. 各杂化轨道中 $s$ 轨道成分分别是 $\alpha_{1}, \alpha_{2}, \alpha_{3}$ 和 $\alpha_{4}$, 它们的加和为 1 . 令 $\psi_{1}, \psi_{2}, \psi_{3}$ 和 $\psi_{4}$ 分别与 $n o^{\prime}, n i, n j$ 和 $n k$ 方向一致. $\psi_{1}$ 与 $\psi_{2}, \psi_{1}$ 和 $\psi_{3}, \psi_{1}$ 与 $\psi_{4}$ 之间的夹角均等于 $\theta_{\sigma \pi}$. 根据杂化轨道之间夹角的余弦公式得到 $\alpha_{2}, \alpha_{3}$ 和 $\alpha_{4} 三$ 者相等. 由此得到两个方程式

$$
\begin{gathered}
\alpha_{1}+3 \alpha_{2}=1, \\
{\left[\frac{\alpha_{1} \alpha_{2}}{\left(1-\alpha_{1}\right)\left(1-\alpha_{2}\right)}\right]^{1 / 2}=-\cos \theta_{\sigma \pi},}
\end{gathered}
$$

将式(5), (6) 代人式(7)得到

$$
\frac{1}{\alpha_{1}}=\left[7.5 \frac{(\operatorname{tg} \phi-\operatorname{tg} \omega)}{\pi}-0.5\right]+0.375 \frac{\operatorname{tg} \omega}{\pi} n,
$$

由式 (8) 可知, 杂化轨道 $\psi_{1}$ 中 $s$ 轨道成分 $\alpha_{1}$ 的倒数与原子簇 $\mathrm{C}_{n}$ 中碳原子数 $n$ 成直线关系, 截 距是 $\left[7.5 \frac{(\operatorname{tg} \phi-\operatorname{tg} \omega)}{\pi}-0.5\right]$, 斜率为 $0.375 \frac{\operatorname{tg} \omega}{\pi}$.

\section{2 计 算 结 果}

在原子簇 $\mathrm{C}_{n}$ 封闭多面体中, 设五边形的角 $2 \phi$ 为 $108^{\circ}$, 六边形的角 $2 \omega$ 是 $120^{\circ}$. 由公式 (5)计算得到 $\theta_{\sigma \pi}$ 值列人表 1.

表 $1 \theta_{\sigma \pi}$ 值

\begin{tabular}{ccccccc}
\hline$C_{n}$ & $\mathrm{C}_{180}$ & $\mathrm{C}_{500}$ & $\mathrm{C}_{1600}$ & $\mathrm{C}_{4500}$ & $\mathrm{C}_{14500}$ & $\mathrm{C}_{4370}$ \\
\hline$\theta_{\sigma \pi}{ }^{\circ}$ & 96.7363 & 93.8532 & 92.2179 & 91.2778 & 90.7391 & 90.4240 \\
\hline
\end{tabular}

由表 1 中 $\theta_{o n}$ 既不等于 $90^{\circ}$, 又不等于 $109.5^{\circ}$ 说明, 具有 $I_{h}$ 对称性未知结构参数的碳 原 子簇 $\mathrm{C}_{n}$ 中碳原子的轨道应采取不等性 $s p^{3}$ 杂化.

由式(6)，(8)计算得到 $\alpha_{1}, \alpha_{2}, \alpha_{3}$ 和 $\alpha_{4}$ 的值列人表 2 .

表 $2 \alpha_{1}$ 和 $\alpha_{2}$ 的值 $\left(\alpha_{3}, \alpha_{4}\right.$ 与 $\alpha_{2}$ 相等 $)$

\begin{tabular}{ccccccc}
\hline $\mathrm{C}_{n}$ & $\mathrm{C}_{180}$ & $\mathrm{C}_{500}$ & $\mathrm{C}_{1000}$ & $\mathrm{C}_{4850}$ & $\mathrm{C}_{14500}$ & $\mathrm{C}_{43700}$ \\
\hline$\alpha_{1}$ & 0.02789 & 0.00907 & 0.00300 & 0.00100 & 0.00033 & 0.00011 \\
$\alpha_{2}$ & 0.32404 & 0.33031 & 0.33233 & 0.33300 & 0.33322 & 0.33330 \\
\hline
\end{tabular}

由 表 2 中 $\alpha_{1}$ 的值可知, $\psi_{1}$ 中 $s$ 轨道的成分 $\alpha_{1}$ 随着 $n$ 的增加而减少. $p$ 轨道的成分 $\left(1-\alpha_{1}\right)$ 随着 $n$ 的增加而增加.

杂化轨道波函数的一般表示式是

$$
\begin{gathered}
\psi_{i}=\sqrt{\alpha_{i}} \psi_{s}+\sqrt{1-\alpha_{i}} \psi_{P_{i}}=\sqrt{\alpha_{i}} \psi_{s}+\sqrt{1-\alpha_{i}}\left(a_{i} \psi_{P_{x}}+b_{i} \psi_{P_{y}}+c_{i} \psi_{P_{2}}\right) \\
=C_{i s} \psi_{s}+C_{i x} \psi_{P_{x}}+C_{i} \psi_{P_{y}}+C_{i z} \psi_{P_{z}},
\end{gathered}
$$


在图 1 中, $n$ 为坐标原点, $n o^{\prime}$ 选为 $z$ 轴, $\psi_{1}$ 与 $z$ 轴方向一致. $\psi_{2}$ 与 $n i$ 方向一致,且在 $x z$ 平面 内. $\psi_{3}$ 和 $\psi_{4}$ 分别与 $n j$ 和 $n k$ 方向相同. 将表 2 中 $\alpha_{i}$ 值代人式 (9), 根据杂化轨道的归一性 和正交性, 计算得到 $a_{i}, b_{i}, C_{i}$ (表 3) 和 $C_{i s}, C_{i x}, C_{i y}, C_{i z}$ (表 4).

表 $3 a_{i}, b_{i}$ 和 $C_{i}$ 的值

\begin{tabular}{|c|c|c|c|c|}
\hline $\mathrm{C}_{n}$ & 杂化轨道 & $a_{i}$ & $b_{i}$ & $C_{i}$ \\
\hline \multirow{4}{*}{$\mathrm{C}_{180}$} & $\psi_{1}$ & 0.00000 & 0.00000 & 1.00000 \\
\hline & $\psi_{2}$ & 0.99310 & 0.00000 & -0.11727 \\
\hline & $\psi_{3}$ & -0.49656 & 0.86005 & -0.11727 \\
\hline & $\psi_{4}$ & -0.49656 & -0.86009 & -0.11727 \\
\hline \multirow{4}{*}{$\mathrm{C}_{540}$} & $\psi_{1}$ & 0.00000 & 0.00000 & 1.00000 \\
\hline & $\psi_{2}$ & - 0.99774 & 0.00000 & -0.06720 \\
\hline & $\psi_{3}$ & -0.49886 & 0.86407 & -0.06720 \\
\hline & $\psi_{4}$ & -0.49886 & -0.86405 & -0.06720 \\
\hline \multirow{4}{*}{$\mathrm{C}_{1620}$} & $\psi_{1}$ & 0.00000 & 0.00000 & 1.00000 \\
\hline & $\psi_{2}$ & 0.99925 & 0.00000 & -0.03869 \\
\hline & $\psi_{3}$ & -0.49962 & 0.86538 & -0.03869 \\
\hline & $\psi_{4}$ & -0.49962 & -0.86535 & -0.03869 \\
\hline \multirow{4}{*}{$\mathrm{C}_{4860}$} & $\psi_{1}$ & 0.00000 & 0.00000 & 1.00000 \\
\hline & $\psi_{2}$ & 0.99975 & 0.00000 & -0.02236 \\
\hline & $\psi_{3}$ & -0.49987 & 0.86581 & -0.02236 \\
\hline & $\psi_{4}$ & -0.49987 & -0.86578 & -0.02236 \\
\hline \multirow{4}{*}{$\mathrm{C}_{14580}$} & $\psi_{1}$ & 0.00000 & 0.00000 & 1.00000 \\
\hline & $\psi_{2}$ & 0.99991 & 0.00000 & -0.01285 \\
\hline & $\psi_{3}$ & -0.49995 & 0.86596 & -0.01285 \\
\hline & $\psi_{4}$ & -0.49995 & -0.86592 & -0.01285 \\
\hline \multirow{4}{*}{$\mathrm{C}_{43740}$} & $\psi_{1}$ & 0.00000 & 0.00000 & 1.00000 \\
\hline & $\psi_{2}$ & 0.99997 & 0.00000 & -0.00742 \\
\hline & $\psi_{3}$ & -0.49999 & 0.86603 & -0.00742 \\
\hline & $\psi_{4}$ & -0.49999 & -0.86601 & -0.00742 \\
\hline
\end{tabular}

表 4 杂化轨道波函数表示式

\begin{tabular}{cccrrr}
\hline $\mathrm{C}_{n}$ & 杂化轨道 & $C_{i}$ & \multicolumn{1}{c}{$C_{i x}$} & \multicolumn{1}{c}{$C_{i y}$} & \multicolumn{1}{c}{$C_{i,}$} \\
\hline \multirow{3}{*}{$C_{180}$} & $\psi_{1}$ & 0.16700 & 0.00000 & 0.00000 & 0.98596 \\
& $\psi_{2}$ & 0.56925 & 0.81650 & 0.00000 & -0.09642 \\
& $\psi_{3}$ & 0.56925 & -0.40826 & 0.70711 & -0.09642 \\
& $\psi_{4}$ & 0.56925 & -0.40826 & -0.70714 & -0.09642 \\
\hline & $\psi_{1}$ & 0.09524 & 0.00000 & 0.00000 & 0.99545 \\
& $\psi_{2}$ & 0.57473 & 0.81650 & 0.00000 & -0.05499 \\
$C_{580}$ & $\psi_{3}$ & 0.57473 & -0.40824 & 0.70711 & -0.05499 \\
& $\psi_{4}$ & 0.57473 & -0.40824 & -0.70710 & -0.05499 \\
\hline & $\psi_{1}$ & 0.05477 & 0.00000 & 0.00000 & 0.99850 \\
& $\psi_{2}$ & 0.57648 & 0.81650 & 0.00000 & -0.03161 \\
$\mathrm{C}_{1520}$ & $\psi_{3}$ & 0.57648 & -0.40824 & -0.70711 & -0.03161 \\
& $\psi_{4}$ & 0.57648 & -0.40824 & -0.70709 & -0.03161 \\
\hline
\end{tabular}


表 4 (续)

\begin{tabular}{|c|c|c|c|c|c|}
\hline $\mathrm{C}_{n}$ & 杂化轨道 & $C_{i s}$ & $C_{i x}$ & $C_{i y}$ & $C_{\mathrm{iz}}$ \\
\hline \multirow{4}{*}{$\mathrm{C}_{4860}$} & $\psi_{1}$ & 0.03162 & 0.00000 & 0.00000 & 0.99950 \\
\hline & $\psi_{2}$ & 0.57706 & 0.81650 & 0.00000 & -0.01826 \\
\hline & $\psi_{3}$ & 0.57706 & -0.40824 & 0.70711 & -0.01826 \\
\hline & $\psi_{4}$ & 0.57706 & -0.40824 & -0.70708 & -0.01826 \\
\hline \multirow{4}{*}{$\mathrm{C}_{14580}$} & $\psi_{1}$ & 0.01817 & 0.00000 & 0.00000 & 0.99983 \\
\hline & $\psi_{2}$ & 0.57725 & 0.81650 & 0.00000 & -0.01049 \\
\hline & $\psi_{3}$ & 0.57725 & -0.40824 & 0.70712 & -0.01049 \\
\hline & $\psi_{4}$ & 0.57725 & -0.40824 & -0.70708 & -0.01049 \\
\hline \multirow{4}{*}{$\mathrm{C}_{43740}$} & $\psi_{1}$ & 0.01049 & 0.00000 & 0.00000 & 0.99994 \\
\hline & $\psi_{2}$ & 0.57732 & 0.81650 & 0.00000 & -0.00606 \\
\hline & $\psi_{3}$ & 0.57732 & -0.40825 & 0.70711 & -0.00606 \\
\hline & $\psi_{4}$ & 0.57732 & -0.40825 & -0.70711 & -0.00606 \\
\hline
\end{tabular}

由表 4 可见, $\psi_{1}$ 中 $p$ 轨道的成分只有 $p_{z}$ 轨道. 当 $n$ 增加时, $\psi_{1}$ 中 $p_{z}$ 轨道的成分亦增加, 且趋近于 1.

\section{参考文献}

[1] 李文莹、黄荣㮏等,化学学报, 1990, 48: 1216.

[2] 唐敖庆、李前树, 中国科学, B辑, 1992, (1): 14.

[3]陈琼、王成瑞, 科学通报, 1993, 38(1): 40.

[4]陈琼、王成瑞,科学通报, 1993, 38(20): 1871. 